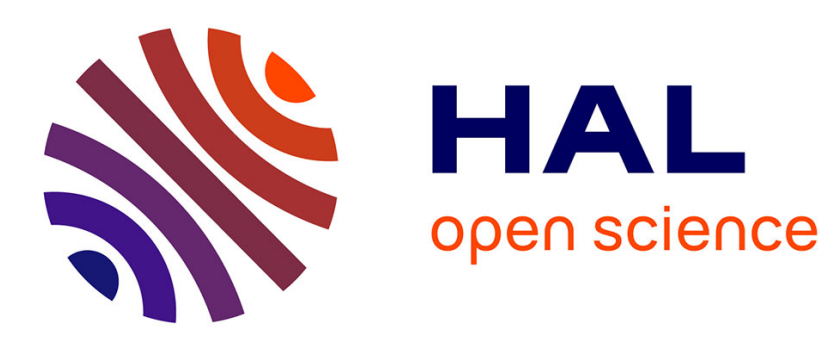

\title{
Charge density wave transport in inorganic linear chain compounds
}

\author{
J. Dumas
}

\section{To cite this version:}

J. Dumas. Charge density wave transport in inorganic linear chain compounds. Revue de Physique Appliquée, 1987, 22 (5), pp.309-319. 10.1051/rphysap:01987002205030900 . jpa-00245546

\section{HAL Id: jpa-00245546 https://hal.science/jpa-00245546}

Submitted on 1 Jan 1987

HAL is a multi-disciplinary open access archive for the deposit and dissemination of scientific research documents, whether they are published or not. The documents may come from teaching and research institutions in France or abroad, or from public or private research centers.
L'archive ouverte pluridisciplinaire HAL, est destinée au dépôt et à la diffusion de documents scientifiques de niveau recherche, publiés ou non, émanant des établissements d'enseignement et de recherche français ou étrangers, des laboratoires publics ou privés. 
Classification

Physics Abstracts

71.45

\title{
Charge density wave transport in inorganic linear chain compounds
}

\author{
J. Dumas \\ C.N.R.S. Laboratoire d'Etudes des Propriétés Electroniques des Solides, Associé à l'Université Scientifique, \\ Technologique et Médicale de Grenoble, B.P. 166, 38042 Grenoble Cedex, France
}

(Reçu le 12 décembre 1986, accepté le 20 janvier 1987)

\section{Article de mise au point}

\begin{abstract}
Résumé. - Un nouveau type de transport collectif par glissement d'une onde de densité de charge sur le réseau cristallin a été mis en évidence ces dernières années dans des conducteurs inorganiques quasi unidimensionnels tels que les trichalcogénures $\mathrm{NbSe}_{3}, \mathrm{TaS}_{3}$, les tétrachalcogénures $\left(\mathrm{TaSe}_{4}\right)_{2} \mathrm{I},\left(\mathrm{NbSe}_{4}\right)_{10 / 3} \mathrm{I}$ et les bronzes bleus de molybdène $\mathrm{A}_{0,3} \mathrm{MoO}_{3}(\mathrm{~A}=\mathrm{K}, \mathrm{Rb}, \mathrm{Tl})$. Nous décrivons les propriétés structurales et électroniques de ces matériaux et leurs étonnantes propriétés de transport non linéaire attachées au mouvement de l'onde : champ électrique seuil, bruit, réponse à une excitation alternative, hystérésis, effets mémoires. Nous rappelons quelques modèles théoriques décrivant la dynamique de l'onde de densité de charge.
\end{abstract}

Abstract. - In quasi-one dimensional inorganic conductors such as the transition metal trichalcogenides $\mathrm{NbSe}_{3}, \quad \mathrm{TaS}_{3}$, the tetrachalcogenides $\left(\mathrm{TaSe}_{4}\right)_{2} \mathrm{I}, \quad\left(\mathrm{NbSe}_{4}\right)_{10 / 3} \mathrm{I}$ and the molybdenum blue bronzes $\mathrm{A}_{0.30} \mathrm{MoO}_{3}(\mathrm{~A}=\mathrm{K}, \mathrm{Rb}, \mathrm{Tl})$ a new collective transport by the macroscopic sliding of a so-called charge density wave through the lattice has been reported. We review the structural and electronic properties of these different classes of materials and describe their intriguing non linear transport properties associated with the charge density wave motion : threshold electric field, noise phenomena, response to ac fields, hysteresis and memory effects. We survey some relevant theoretical models for the dynamics of the charge density wave.

\section{Introduction.}

As a consequence of their anisotropic crystal structure, some materials behave as if they had less than three dimensions. Among the quasi-two dimensional conductors examples are the dichalcogenides $\mathrm{TaS}_{2}$, $\mathrm{TaSe}_{2}$ [1], the purple molybdenum bronzes $\mathrm{A}_{0.9} \mathrm{Mo}_{6} \mathrm{O}_{17}$ [2] $(\mathrm{A}=\mathrm{Li}, \mathrm{Na}, \mathrm{K}), \mathrm{TlMo}_{6} \mathrm{O}_{17}$ [3] and the molybdenum oxides $\mathrm{Mo}_{4} \mathrm{O}_{11}[2]$. Among the quasi-one-dimensional compounds, one finds the organic conductors [4] such as TTF-TCNQ or (TMTSF $)_{2} \mathrm{X}\left(\mathrm{X}=\mathrm{PF}_{6}, \mathrm{ClO}_{4}\right)$ and inorganic conductors such as the transition metal trichalcogenides $\mathrm{NbSe}_{3}, \mathrm{TaS}_{3}$, the tetrachalcogenides $\left(\mathrm{TaSe}_{4}\right)_{2} \mathrm{I}$, $\left(\mathrm{NbSe}_{4}\right)_{10 / 3} \mathrm{I}$ [5] and a completely different family, the molybdenum blue bronzes $\mathrm{A}_{0.30} \mathrm{MoO}_{3}$ $(\mathrm{A}=\mathrm{K}, \mathrm{Rb}, \mathrm{Tl})[6,7]$. These different classes of materials offer exciting opportunities to condensed matter physicists and chemists to study the concept of « charge density wave».

These compounds are unstable towards lattice distortions of wave vector $2 K_{\mathrm{F}}$ where $K_{\mathrm{F}}$ is the wave vector at the Fermi energy; they undergo a phase transition called Peierls transition [8] associated with the onset of a «charge density wave ». The ground state is characterized by a periodic lattice distortion $u(x)$ coupled to a spatial periodic modulation $\rho(x)$ of the concentration of conduction electrons called charge density wave (CDW).

In a one dimensional case the resulting configuration can be written : $\rho(x)=A \cos \left(2 K_{\mathrm{F}} x+\phi\right)$ where $A$ is the amplitude and $\phi$ the phase of the CDW. The associated atomic lattice displacement is : $u(x)=U_{0} \sin \left(2 K_{\mathrm{F}} x+\phi\right)$ where $U_{0}$ is much smaller than a lattice distance $\left(U_{0}<0.1 \AA\right)$. Since the wavelength $\lambda=2 \pi / 2 K_{\mathrm{F}}$ is determined by the Fermi surface, it will usually be incommensurate with the underlying lattice, that is $\lambda$ will not equal a rational multiple of a lattice translation. When the concentration of electron per atom in the filled band is a rational number, the $\mathrm{CDW}$ is commensurate. The CDW is a one-dimensional modulation of both the electron density and of the structure along the direction of highest conductivity. 
Fröhlich [9] has shown that, when $2 K_{\mathrm{F}}$ is incommensurate with the lattice, the phase $\phi$ of the CDW with respect to the lattice is arbitrary and a current could be carried, not by the motion of individual electrons, but by the sliding of the CDW at no cost in energy. This collective transport of electrical current by a moving CDW would lead to superconductivity. However, in real crystals impurities or defects destroy the translational invariance of the $\mathrm{CDW}$ and pin it to the lattice. It is by now well established that pinning forces play a crucial role in electrical properties. The CDW has to overcome potential barriers before the motion begins. The CDW can be depinned by a very weak electric field larger than a critical value $E_{\mathrm{t}}$, the so-called threshold field. Above $E_{\mathrm{t}}$, a rich variety of non linear transport phenomena are found : they include broad band noise, periodic voltage oscillations, metastability phenomena. This new transport mechanism leads also to anomalous frequency dependent conductivity and anomalous response to combined ac and dc fields.

Since the discovery in 1976 by Monceau et al. [10] of CDW transport in the linear chain compound $\mathrm{NbSe}_{3}$ an enormous interest in this field has emerged. Motivated by the possibility that other systems with a high conductivity axis may also provide a realization of the Fröhlich conductivity, it has been shown later, that this new effect appeared also in $\mathrm{TaS}_{3}$ [11], $\left(\mathrm{TaSe}_{4}\right)_{2} \mathrm{I}$ [12], $\left(\mathrm{NbSe}_{4}\right)_{10 / 3} \mathrm{I}$ [13] and in the blue bronzes $\mathrm{A}_{0.30} \mathrm{MoO}_{3}(\mathrm{~A}=\mathrm{K}, \mathrm{Rb}, \mathrm{Tl})$ $[6,7]$. CDW is now an exciting problem in many body physics both theoretically and experimentally. Several international conferences have been devoted to this field and the dramatic increase of the number of papers on CDW's shows the vitality of this subject [14].

In this review, we give in section 1 a description of the Peierls instability; in section 2 we describe the crystal structures and the Peierls transitions of the materials where non linear CDW transport occurs; in section 3 we report some unusual phenomena which give evidence for CDW conductivity; in section 4 we survey some theoretical models which have been advanced to account for this new type of conductivity.

\section{Peierls instability.}

Peierls has shown more than thirty years ago [8] that a one dimensional conducting chain of uniformly spaced atoms is unstable at $0 \mathrm{~K}$ against a periodic distortion with wavevector $\pm 2 K_{\mathrm{F}}$. The gain in electronic energy is always larger than the elastic energy required to distort the lattice. The lowering of energy results from the decrease in energy of the occupied states just below the Peierls gap ; the states which are raised in energy just above the gap are unoccupied. Figure 1 shows the band structure


Fig. 1. - Density of states $\varepsilon(K)$ of a one-dimensional system : a) undistorted state $T>T_{\mathrm{P}}$; b) distorted state $T<T_{\mathrm{P}}$; c) Temperature dependence of the Peierls gap $2 \Delta$.

$\varepsilon(K)$ of a quasi-one dimensional conductor above and below the Peierls transition temperature $T_{\mathrm{P}}$.

The gap which opens at the Fermi energy is proportional to the amplitude of the distortion. The temperature dependence of the gap $2 \Delta$ is illustrated in figure 1c. Within mean field theory, the excitation of electrons across the gap reduces the size of $2 \Delta$ to zero at $T_{\mathrm{P}}[15]$.

In a one dimensional conductor the Peierls transition is a metal-to-semiconductor transition; in a quasi two-dimensional conductor the transition is associated with partial opening of gaps at the Fermi surface and is a metal-to-metal transition. The Peierls transition has been investigated in several organic and inorganic low dimensional conductors: polyacetylene, organic charge transfert salts, transition metal di, tri and tetrachalcogenides, blue and purple bronzes and molybdenum oxides $\mathrm{Mo}_{4} \mathrm{O}_{11}$. In the organic salt TTF-TCNQ non linear conductivity has been reported recently [16].

An instability occurs if the response $\Delta \rho(q)$ of the electron gas to a small perturbing potential $V(q)$ becomes macroscopic. If $\Delta \rho(q)=X(q) V(q)$ where $X(q)$ is the response function, a static distortion occurs if $X(q) \rightarrow \infty . X(q)$ is given by :

$$
X(q) \propto \sum_{K} \frac{f_{K}\left(1-f_{K+q}\right)}{\varepsilon_{K+q}-\varepsilon_{K}}
$$

where $\varepsilon_{\mathbf{K}}$ is the energy of states with wavevector $K$ and $f_{K}$ the Fermi occupation factor. $X(q)$ is large for a given $q$ which connects many filled states to empty states of the same energy. This property is called nesting of the Fermi surface. A Fermi surface with plane sections will produce a $X(q)$ which diverges 
when $q$ spans the Fermi surface. In a quasi-one dimensional case, the Fermi surface consists of two parallel planes separated by a distance $2 K_{\mathrm{F}}$ and allows a strong divergence of $X(q)$.

\section{Crystal structures and Peierls transitions.}

Direct evidence for the onset of a CDW state comes from electron diffraction microscopy or from X-ray scattering. Each main Bragg peak has a series of satellite peaks around it ; the satellite peaks have a very weak intensity, $\approx 10^{-3}$ or less of the intensity of the strongest Bragg peak. Up to now it is only in the two dimensional compound $\mathrm{TaS}_{2}$ that the CDW state has been observed recently in the real space by scanning tunnelling microscopy [17]. In the tri- and tetrachalcogenides the expected satellite spots in the CDW state have been observed by electron microscopy [18-22]. However, in the case of the blue bronzes, the satellite peaks have been observed only by X-ray scattering experiments [23], since these bronzes are extremely sensitive to electron irradiation which creates permanent damage [24].

2.1 TRICHALCOGENIDES. - The trichalcogenide $\mathrm{NbSe}_{3}$ was first synthetized by chemical vapor transport at $T \approx 700{ }^{\circ} \mathrm{C}$ by Meerschaut and Rouxel in 1975 [25]. $\mathrm{NbSe}_{3}$ appears as needle shaped single crystals, several mm long, somewhat flexible along the needle axis, and with a typical cross section of $50 \mu^{2}$.

The structure consists of chains along the b-axis of trigonal prisms of $\mathrm{Se}$ with transition metal approximately at the centre of each chalcogen prism. The chains are linked in the c-direction by interchain $\mathrm{Nb}$ Se bonds (Fig. 2). The conductivity along the chains is large $\sigma \approx 10^{4} \Omega^{-1} \mathrm{~cm}^{-1} . \mathrm{NbSe}_{3}$ is not highly anisotropic, the ratio between $\sigma_{\| b}$ and $\sigma_{\perp b}$ is $\approx 10$ and the CDW onset suppresses only part of the Fermi Surface. Two CDW transitions occur, one at $T_{1}=145 \mathrm{~K}$, the other one at $T_{2}=59 \mathrm{~K}$ leaving the system metallic. These two independent transitions are marked in the resistivity as a function of temperature by two anomalies [26] as shown in figure 3 . These anomalies can be suppressed by high electric fields or high frequencies [27]. The components of

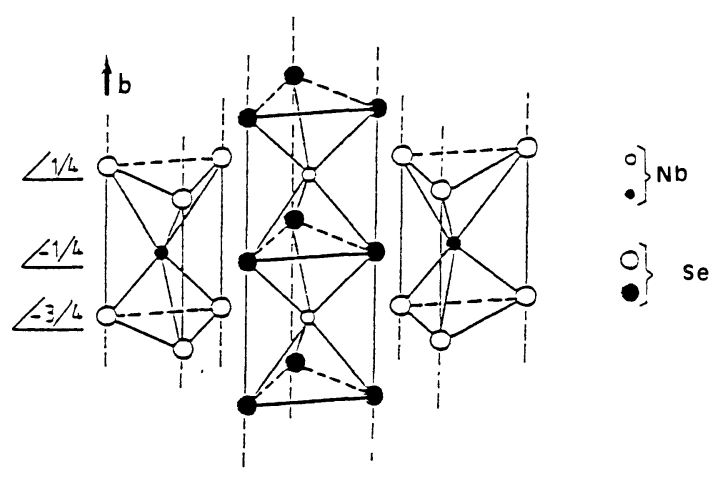

Fig. 2. - Crystal structure of $\mathrm{NbSe}_{3}$ (from Ref. [10]).

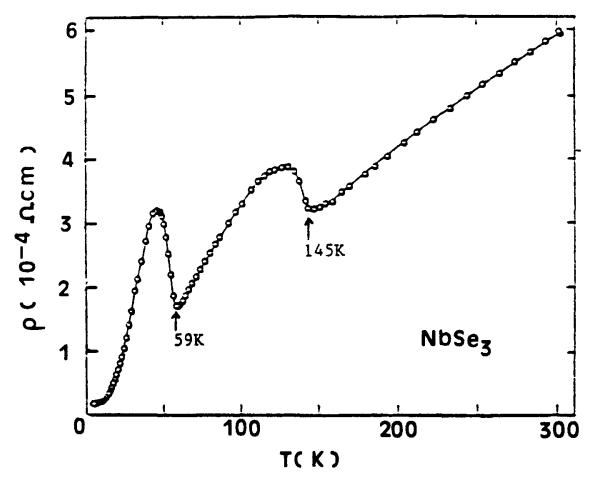

Fig. 3. - Resistivity as a function of temperature of $\mathrm{NbSe}_{3}$; current along the fiber axis (from Ref. [26]).

Table I. - Parameters of the Peierls transitions, crystal symmetry and components of wavevector of the Peierls distortion.

\begin{tabular}{|c|c|c|c|c|c|c|c|c|c|}
\hline & $T_{\mathrm{p}}(\mathrm{K})$ & $\begin{array}{c}\text { Anisotropy } \\
\text { factor } \frac{\sigma_{\|}}{\sigma_{\perp}}\end{array}$ & $\begin{array}{l}\text { Resistivity } \\
\text { at } 300 \mathrm{~K}\end{array}$ & $\begin{array}{l}\text { Threshold } \\
E_{\mathrm{t}}(\mathrm{V} / \mathrm{cm})\end{array}$ & Symmetry & $\begin{array}{c}q_{a} \\
\left(a^{*} \text { unit }\right)\end{array}$ & $\begin{array}{c}q_{b} \\
\left(b^{*} \text { unit }\right)\end{array}$ & $\begin{array}{c}q_{c} \\
\left(c^{*} \text { unit) }\right.\end{array}$ & $\begin{array}{l}\text { Refe- } \\
\text { rences }\end{array}$ \\
\hline $\mathrm{NbSe}_{3}$ & $\begin{array}{r}144 \\
59\end{array}$ & $\sim 10$ & $6 \times 10^{-4}$ & $\begin{array}{l}0.1 \\
0.01\end{array}$ & Monoclinic & $\begin{array}{l}0 \\
0.5\end{array}$ & $\begin{array}{l}0.2412 \\
0.2604\end{array}$ & $\begin{array}{l}0 \\
0.5\end{array}$ & {$[18]$} \\
\hline $\mathrm{O}-\mathrm{TaS}_{3}$ & 215 & $\sim 100$ & $5 \times 10^{-4}$ & 2 & Orthorombic & $1 / 2$ & $1 / 8$ & 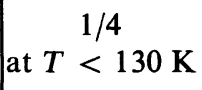 & [19] \\
\hline $\mathrm{m}-\mathrm{TaS}_{3}$ & 240 & & $5 \times 10^{-4}$ & & Monoclinic & 0 & 0.254 & 0 & [20] \\
\hline$\left(\mathrm{TaSe}_{4}\right)_{2} \mathrm{I}$ & 263 & & $1.5 \times 10^{-3}$ & 2 & Tetragonal & 1 & 0 & 0.943 & {$[21]$} \\
\hline$\left(\mathrm{NbSe}_{4}\right)_{10 / 3} \mathrm{I}$ & 285 & & $10^{-2}$ & 1 & Tetragonal & 0 & 0 & 0.487 & {$[22]$} \\
\hline $\begin{array}{l}\mathrm{A}_{0.3} \mathrm{MoO}_{3} \\
(\mathrm{~A}=\mathrm{K}, \mathrm{Rb})\end{array}$ & 180 & $\sim 100$ & $10^{-3}$ & 0.05 & Monoclinic & 0 & $\begin{array}{c}0.250 \\
T<100 \mathrm{~K}\end{array}$ & 0.5 & {$[23]$} \\
\hline
\end{tabular}


the wavevector of the distortion are temperature independent and are listed in table I which gives also data for other CDW materials.

While $\mathrm{NbSe}_{3}$ is not very anisotropic and remains metallic below $T_{2}, \mathrm{TaS}_{3}$ has been found to be more anisotropic, $\sigma_{\|} / \sigma_{\perp} \approx 100$. The phase transition is a metal-to-semiconductor transition and the Fermi Surface is completely destroyed. In $\mathrm{TaS}_{3}$, weaker interchain bonding allows the occurrence of two polytypes, orthorhombic and monoclinic. Orthorhombic $\mathrm{TaS}_{3}$ has a Peierls transition at $215 \mathrm{~K}$ [11]. The resistivity of $0-\mathrm{TaS}_{3}$ as a function of temperature is shown in figure 4 . The wavevector of the distortion is temperature dependent and locks to $0.25 c^{*}$ at $\approx 130 \mathrm{~K}$ [28]. Monoclinic $\mathrm{TaS}_{3}$ is isotype with $\mathrm{NbSe}_{3}$ and shows two transitions at $T_{1}=$ $240 \mathrm{~K}$ and $T_{2}=160 \mathrm{~K}$ respectively [25].

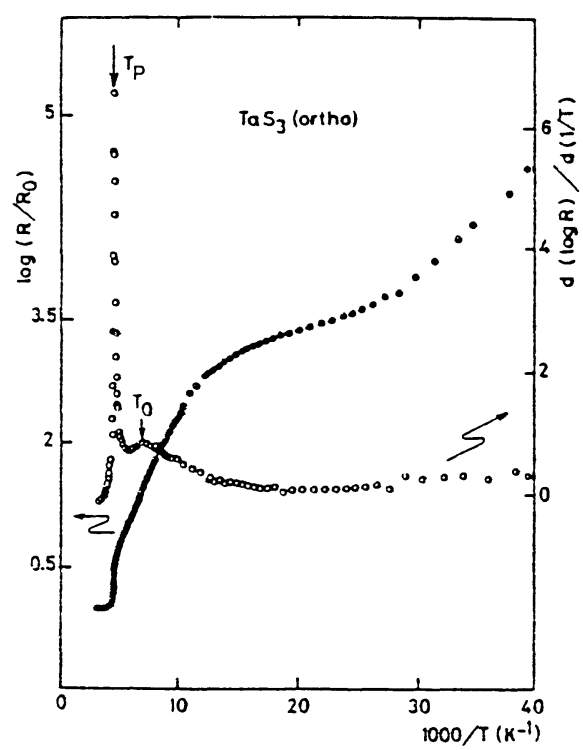

Fig. 4. - Resistivity and its logarithmic derivative as a function of reciprocal temperature of orthorombic $\mathrm{TaS}_{3}$. $T_{\mathrm{P}}$ is the Peierls transition temperature and $T_{0}$ is the commensurate-incommensurate transition temperature (from Ref. [5]).

2.2 Tetrachalcogenides. - The large class of tetrachalcogenides have the general formula $\left(\mathrm{MX}_{4}\right)_{n} \mathrm{Y}$ where $\mathrm{M}=\mathrm{Nb}, \mathrm{Ta}, \mathrm{X}=\mathrm{S}, \mathrm{Se}, \mathrm{Y}=\mathrm{Cl}$, $\mathrm{Br}, \mathrm{I}$ and $n=2,3,4,10 / 3$. They were synthetized for the first time by Meerschaut et al. [21] by chemical vapour transport by heating the elements in sealed quartz tubes at $500-600{ }^{\circ} \mathrm{C}$. $\left(\mathrm{MX}_{4}\right)_{n} \mathrm{Y}$ crystals are platelets a few $\mathrm{mm}$ long and with a cross section of a few tenths of $\mathrm{mm}^{2}$.

The structure of $\left(\mathrm{TaSe}_{4}\right)_{2} \mathrm{I}$ consists of chains of $\mathrm{MSe}_{4}$ along the $c$ axis, the iodine atoms are located in the channels between the chains. The interchain distance $\mathbf{M}-\mathbf{M}$ is much larger than the intrachain distance. In each $\mathrm{TaSe}_{4}$ chain, the Ta atom is located


Fig. 5. - Crystal structure of $\left(\mathrm{TaSe}_{4}\right)_{2} \mathrm{I}$ (from Ref. [21]).

at the centre of a rectangular antiprism of $8 \mathrm{Se}$ atoms (Fig. 5).

$\left(\mathrm{TaSe}_{4}\right)_{2} \mathrm{I}$ shows a Peierls transition at $263 \mathrm{~K}$ from a metallic to a semiconducting state. The electrical anisotropy is enhanced by the presence of I atoms ; $\left(\mathrm{TaSe}_{4}\right)_{2} \mathrm{I}$ is more anisotropic than the trichalcogenides. $\left(\mathrm{NbSe}_{4}\right)_{10 / 3} \mathrm{I}$ has a slightly different crystal structure and shows a Peierls transition at $285 \mathrm{~K}$.

2.3 Molybdenum Blue BRONZes. - The molybdenum bronzes have the general formula $\mathrm{A}_{x} \mathrm{M}_{y} \mathrm{O}_{z}$ where $\mathrm{A}$ is an alkali metal. The alkali atom donates its outer electron to the conduction band of the transition metal. They belong to the large class of transition metal oxides which often exhibit metalsemiconductor transitions or CDW instabilities.

While the quasi-one dimensional blue bronze $\mathrm{A}_{0.30} \mathrm{MoO}_{3}(\mathrm{~A}=\mathrm{K}, \mathrm{Rb}, \mathrm{Tl})$ shows a metal insulator transition at $180 \mathrm{~K}$ [2] the quasi-two dimensional purple bronze $\mathrm{K}_{0.9} \mathrm{Mo}_{6} \mathrm{O}_{17}$ shows a metal-to-metal transition at $120 \mathrm{~K}(2)$ due to a CDW instability and $\mathrm{Li}_{0.9} \mathrm{Mo}_{6} \mathrm{O}_{17}$ becomes superconductor below $1.9 \mathrm{~K}[30]$.

Single crystals of blue bronze $\mathrm{K}_{0.30} \mathrm{MoO}_{3}$ were synthetized more than twenty years ago by Wold et al. [31] by electrocrystallization of a molten mixture $\mathrm{K}_{2} \mathrm{MoO}_{4}-\mathrm{MoO}_{3}$ at $550{ }^{\circ} \mathrm{C}$. They appear as platelets of typical size $5 \mathrm{~mm} \times 2 \mathrm{~mm} \times 1 \mathrm{~mm}$. Samples as large as $6 \mathrm{~cm}^{3}$ have been grown recently by this method [14e]. The metal-insulator transition at $180 \mathrm{~K}$ was discovered in 1965 by Bouchard et al. [32]. It was only in 1983 that the transition was shown to be a Peierls transition by Pouget et al. [23] and that CDW transport was reported by Dumas et al. [33].

The crystal structure is monoclinic [34]. It can be viewed as a quasi-two dimensional one but the electronic properties are quasi one-dimensional. The structure is built upon sheets of $\mathrm{MoO}_{6}$ octahedra 


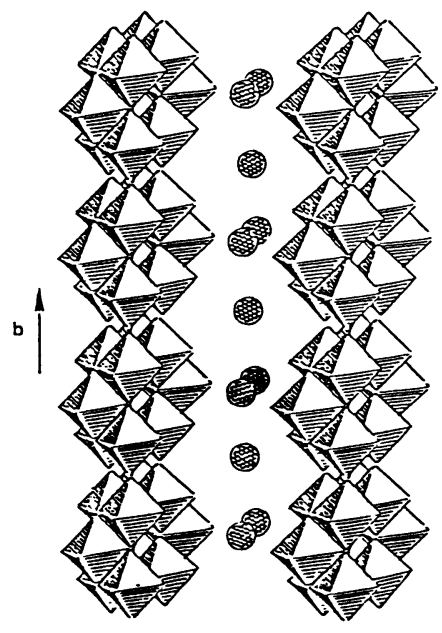

Fig. 6. - Crystal structure of the blue bronze $\mathrm{K}_{0.30} \mathrm{MoO}_{3}$ showing infinite slabs of $\mathrm{MoO}_{6}$ separated by the alkali ions $(\bullet)$ and the infinite chains of $\mathrm{MoO}_{6}$ parallel to the $b$ axis (from Ref. [34]).

sharing edges. The $\mathrm{MoO}_{6}$ octahedra are separated by the alkali ions. The structure bears some analogy with that of $\left(\mathrm{TaSe}_{4}\right)_{2} \mathrm{I}$. The structure can also be viewed as made of infinite chains of $\mathrm{MoO}_{6}$ along the monoclinic $b$-axis sharing corners (Fig. 6). There are three independent Mo sites and two of them are involved in the infinite chains. All the alkali sites are occupied. The CDW is incommensurate along the high conductivity monoclinic $b$-axis ; the wavevector $q_{b}$ is temperature dependent down to $\approx 100 \mathrm{~K}$; below $100 \mathrm{~K}, q_{b}$ is temperature independent and extremely close to the commensurate value $0.25[23]$.

Figure 7 shows the resistivity as a function of temperature for $\mathrm{Rb}_{0.3} \mathrm{MoO}_{3}$ which is isotype of $\mathrm{K}_{0.3} \mathrm{MoO}_{3}$. The resistivity is one order of magnitude

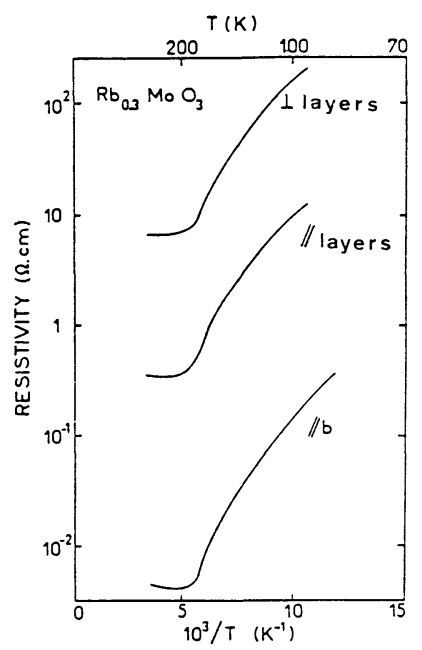

Fig. 7. - Resistivity as a function of reciprocal temperature of $\mathrm{Rb}_{0.30} \mathrm{MoO}_{3}$ for different crystallographic orientations (from Ref. [6]). larger in the plane of the layers than along the high conductivity $b$-axis. Across the layers, $\rho$ is more than three orders of magnitude larger. The blue bronzes are therefore extremely anisotropic materials. The low dimensional electronic properties can be understood easily with the crystal structure.

\section{Charge density wave transport.}

3.1 NON LINEAR CONDUCTIVITY. - The competition between pinning forces and the external applied field is responsible for non linear phenomena. The collective motion of the CDW provides an additional contribution to the conductivity resulting in a non linear current.

Voltage characteristic can be observed by measuring voltage-current characteristics $V(I)$ or $\sigma(E)$ or the derivative $\mathrm{d} V / \mathrm{d} I$ vs. $I$ by lock-in detection. The threshold field $E_{\mathrm{t}}$ for the onset of non linearity is defined when $\mathrm{d} V / \mathrm{d} I$ starts to decrease. Two or four probes configuration for the leads are used. Considerable care in the preparation of the electrical contacts has to be taken to obtain a current density in the sample as homogeneous as possible.

Non linear conductivity above a well defined electric threshold field $E_{\mathrm{t}}$ was first reported by Fleming and Grimes [35] in $\mathrm{NbSe}_{3}$. Below $E_{\mathrm{t}}$, the conductivity is Ohmic. $E_{\mathrm{t}}$ is as low as $5 \mathrm{mV} / \mathrm{cm}$. Examples of $V(I)$ and $\mathrm{d} V / \mathrm{d} I$ vs. $I$ curves are given in figure 8 for $\mathrm{TaS}_{3}$ [28]. The onset of non linearity can be smooth or marked by an abrupt switching from the linear to the non linear state depending on the samples or the temperature. In the case of $\mathrm{K}_{0.30} \mathrm{MoO}_{3}$ [6], precursor voltage spikes of very low frequency $(\approx 1 \mathrm{~Hz})$ occur just below threshold in samples exhibiting a switching at $E_{\mathrm{t}}$ as shown in

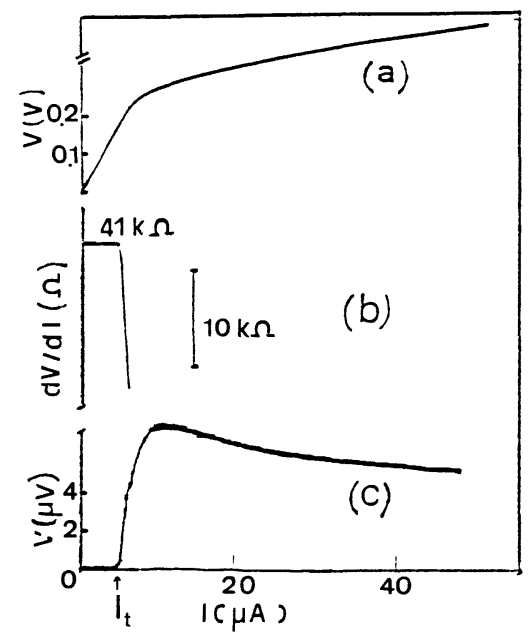

Fig. 8. - a) Voltage vs. current characteristic ; b) differential resistance; c) broad band noise voltage versus applied current $I$ in ortho-TaS $\mathrm{T}_{3}$ at $T=142.4 \mathrm{~K}$ (from Ref. [28]). $E_{\mathrm{t}}=R I_{\mathrm{t}} / l, l$ is the distance between voltage contacts. 


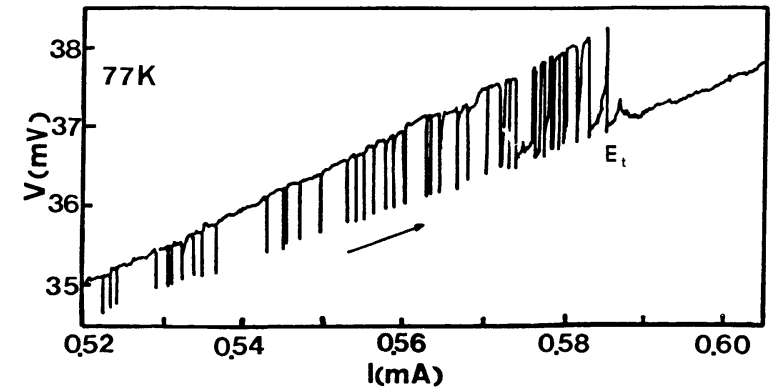

Fig. 9. - Voltage vs. current characteristic of $\mathrm{K}_{0.30} \mathrm{MoO}_{3}$ showing precursor pulses (from Ref. [6]).

figure 9. $V(I)$ curves usually show an hysteretic behaviour. The temperature dependence of the threshold is controversial at the moment in the case of the blue bronze, especially above $100 \mathrm{~K}$ temperature above which the wavevector is temperature dependent. Doping with impurities leads to an increase of $E_{\mathrm{t}}$. According to the theory of Lee and Rice discussed in section 4 pinning centres can be classified as strong and weak. In the weak pinning case, the phase of the CDW is adjusted over a distance larger than the mean impurity separation while in the strong pinning case the phase is adjusted at each impurity site.

The non-linear conductivity is due to the motion of the CDW since no structural change in the CDW appears with the applied field [36]. Moreover, this effect is collective since the energy provided by the electric field over a distance 1 has to be larger than $k T$; writing $e E_{\mathrm{t}} l>k T$ one finds $l \approx 10^{4}$ lattice constants.

3.2 BROAD BAND NOISE AND PERIODIC VOLTAGE OSCILLATIONS. - Just above threshold a broad band noise voltage, orders of magnitude larger than that observed in ordinary semiconductors is observed as illustrated in figure 9. An astonishing and one of the most discussed phenomenon associated with CDW transport is the observation of coherent voltage oscillations, also called "narrow band noise " superimposed on the broad band noise. These oscillations generated above $E_{\mathrm{t}}$ were first discovered by Fleming and Grimes [35] in $\mathrm{NbSe}_{3}$ by Fourier analysis of the noise voltage at a given fixed current. The noise spectrum consists of sharp peaks in the Fourier spectrum with one fundamental frequency and its harmonics as shown in figure 10. Voltage oscillations can also be observed directly by measuring the response to a current pulse. The oscillations are superimposed on the dc voltage response. The frequency increases linearly with the excess CDW current $I_{\mathrm{CDW}}$ defined as $I_{\mathrm{CDW}}=I_{\text {mes }}-I_{\text {ohmic }}$ where $I_{\text {mes }}$ is the measured current and $I_{\text {ohmic }}=V / R$ is the current extrapolated from the Ohmic portion of the $V$-I curve.

If the voltage oscillation is associated with the



Fig. 10. - Fourier spectrum of the noise voltage in $\mathrm{NbSe}_{3} ; E_{\mathrm{t}}=80.4 \mathrm{mV} / \mathrm{cm}$ (from Ref. [37]).

time required for the CDW to travel of one wavelength $\lambda$ then $f=V_{\mathrm{CDw}} / \lambda$ where $V_{\mathrm{CDW}}$ is an average velocity and since $j_{\mathrm{CDW}}=n e v_{\mathrm{CDw}}$ one finds [37] $f / j_{\mathrm{CDW}}=1 / n e \lambda$. Taking values of $\lambda$ deduced from X-ray data, the value of $n$ which is found is in good agreement with that obtained from other transport measurements.

Convincing evidence for the motion of the CDW above $E_{\mathrm{t}}$ has been found in $\mathrm{NbSe}_{3}$ [38] and in the blue bronzes [39] by NMR experiments on the Nb and $\mathrm{Rb}$ nuclei respectively with an applied current in the sample larger than the threshold current. The observed motional narrowing of the NMR lineshape is due to the modulation of the electric field gradient on the $\mathrm{Nb}$ or $\mathrm{RB}$ sites when the CDW slides, figure 11.

The exact origin of the periodic voltage oscillations is still controversial : is it generated in the bulk of the sample or near the end contacts where the carriers condensed in the CDW are converted into normal carriers? Verma et al. [40] have found that the

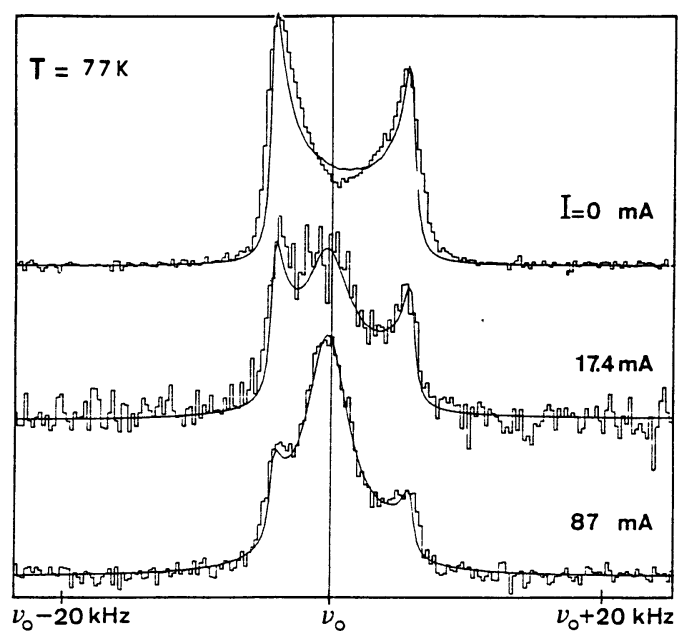

Fig. 11. - Motional narrowing of the ${ }^{87} \mathrm{Rb}$ NMR line shape as a function of the current in $\mathrm{Rb}_{0.3} \mathrm{MoO}_{3}$. $I_{\mathrm{T}} \approx 8 \mathrm{~mA}$. (Segransan et al. Ref. [39]). 
frequency observed in the Fourier spectrum was split into two frequencies by application of a thermal gradient $\Delta T$ along a sample of $\mathrm{NbSe}_{3}$. For $\Delta T \neq 0$ each frequency has been attributed to one end contact. These authors have therefore concluded that the noise was generated at the end contacts. However, additional splittings of the frequency have been found by Brown et al. [41] when one increases $\Delta T$. In the case of the blue bronze, the application of a thermal gradient did not lead to a splitting of the frequency [42]. Other experiments using non perturbing contact potentials show that most of the narrow band noise is generated in the bulk [43].

3.3 FREQUENCY DEPENDENT CONDUCTIVITY. The ac dynamics also shows that the CDW motion is collective. If one assumes single particle excitations at $\approx 10 \mathrm{MHz}$, frequency range where $\sigma$ is strongly frequency dependent, the energy is $\approx 10^{-7} \mathrm{eV}$. Near the Peierls transition temperature the energy per degree of freedom is $k T \approx 10^{-2} \mathrm{eV}$ several orders of magnitude larger than the energy given above. Therefore, the number of thermal degrees of freedom in CDW materials is much smaller than the number of particles. In the CDW state, $\sigma_{\mathrm{ac}}$ is frequency dependent at microwave frequencies and much larger than $\sigma_{\mathrm{dc}}$. This is in contrast with a classical metal where one expects a frequency dependent conductivity only in the optical region.

The temperature dependence of $\sigma_{\mathrm{ac}}$ as a function of temperature is illustrated in figure 12 for $\mathrm{TaS}_{3}$ [14a]. $\operatorname{Re} \sigma$ increases with $\omega$ and $\operatorname{Im} \sigma$ has a maximum in the region where $\operatorname{Re} \sigma$ depends strongly on $\omega$. From the experimental data a giant dielectric constant $\varepsilon=\operatorname{Im} \sigma / \omega$ is found : $\varepsilon \approx 10^{7}$.

In the non linear regime where the CDW is depinned a change in $\sigma_{d c}$ is found as a result of an applied ac field of arbitrary frequency and amplitude.

Monceau et al. [37] have observed peaks in the differential conductivity when an external rf field is

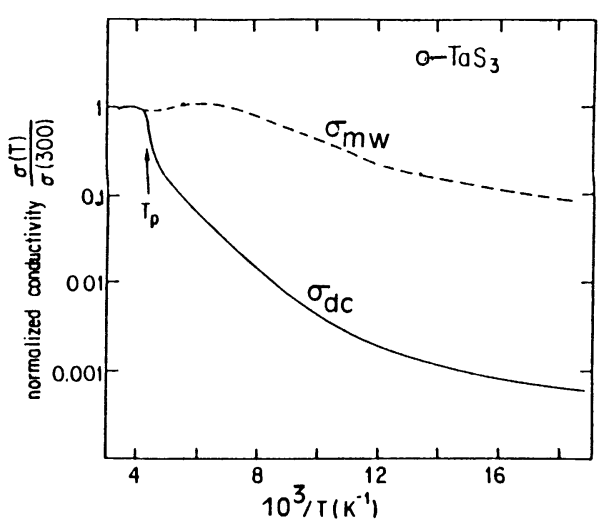

Fig. 12. - Microwave conductivity $\sigma_{\mathrm{mw}}$ and dc conductivity $\sigma_{\mathrm{dc}}$ of ortho-TaS $\mathrm{Ta}_{3}$ as a function of reciprocal temperature (from Ref. [14a]). applied. They correspond to steps in $V-I$ curves, and are due to interference effects between the applied ac field and the dc induced voltage oscillations. Typical results are shown in figure 13. They are analogous to those found in Josephson junctions.

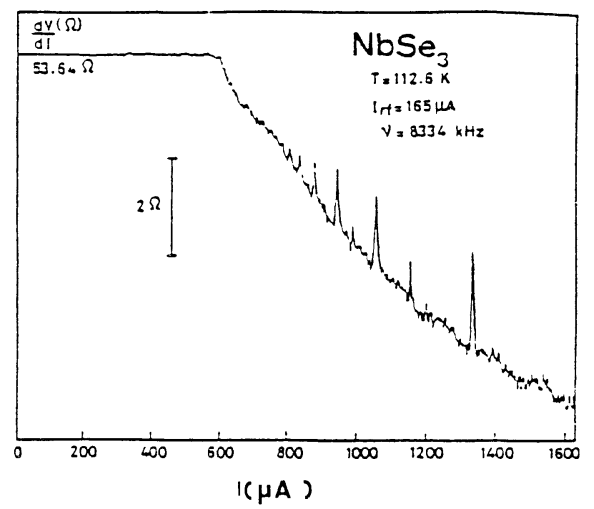

Fig. 13. - Differential conductivity $\mathrm{d} V / \mathrm{d} I$ as a function of a bias current with an applied ac field (from Ref. [37]).

3.4 HYSTERESIS AND MEMORY EFFECTS. - Several experiments [14e] indicate that a CDW is a deformable system and that the non linear transport depends on the past electrical and thermal history of the sample. For example, in $\mathrm{Rb}_{0.30} \mathrm{MoO}_{3}$, very low frequency voltage oscillations $(\approx 1 \mathrm{~Hz})$ are generated well above threshold when the sample is cooled rapidly from $300 \mathrm{~K}$ to $77 \mathrm{~K}$ with an applied current as it can be seen in figure 14. It appears that the transition between pinned and current carrying state is not instantaneous. The switching and hysteresis at threshold is associated with long times required to establish the non linear state. The random distribution of pinning centres is expected to lead to metastable states [44] associated with domains of the CDW which are less pinned than others. The randomness in these $\mathrm{CDW}$ materials suggest some

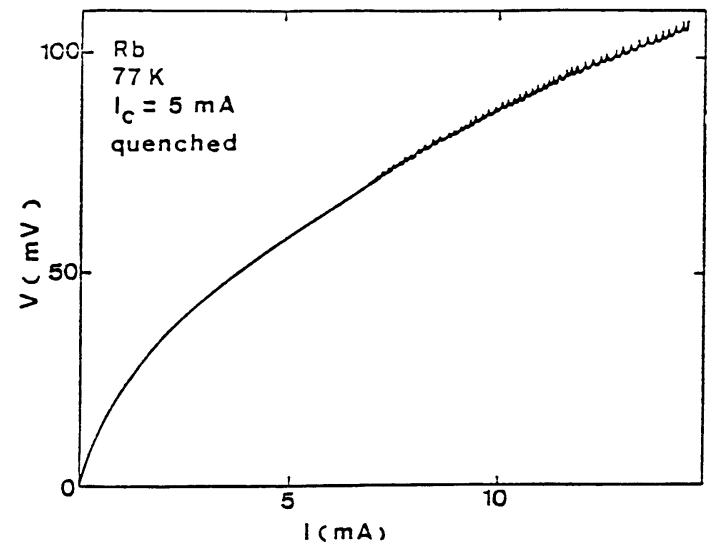

Fig. 14. - Voltage vs. current characteristic of $\mathrm{Rb}_{0.30} \mathrm{MoO}_{3}$ after quenching with an applied current $I_{\mathrm{c}}=5 \mathrm{~mA}$ (from Ref. [6]). 
similarities with spin glasses where a broad distribution of relaxation times is involved. As in glassy materials, $\sigma_{\mathrm{ac}}$ can also be described at low frequency $(<500 \mathrm{MHz})$ by the empirical formula $\sigma(\omega)=$ $A\left(i \omega / \omega_{0}\right)^{\alpha} \alpha<1$.

Among the remarkable effects due to the existence of metastable states is the pulse sign memory effect [45] illustrated in figure 15 . When voltage pulses have opposite polarities a transient enhancement of the non linear conduction («overshoot») is observed.

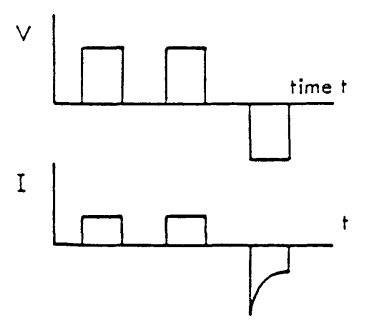

Fig. 15. - Pulse sign memory effect (from Ref. [45]).

Structural evidence for the metastability of the CDW has been given by Tamegai et al. [45] and by Fleming et al. [47] in the case of the blue bronze. Fleming et al. have found that the width of a superlattice peak was broader after an electric field has been applied during a short time. By cooling with an applied field $E>E_{\mathrm{t}}$, the transverse width of the peak was increased. These results indicate a loss of long range order in the direction transverse to the incommensurate component of the wavevector. Another illustration of the relaxation of the $\mathrm{CDW}$ is the generation of a thermally stimulated depolarization current, as found by Cava et al. [48] in the blue bronze, obtained after cooling the sample with an applied electric field. By heating, a weak thermally stimulated current is generated, the sample acting in this situation as a current source. On the other hand, Kriza et al. [49] have found that the dielectric relaxation in the blue bronze obeys a stretched exponential behaviour $P(t)=P_{0} \exp -(t / \tau)^{\alpha}$ where $P(t)$ is the time dependent polarization. The stretched exponential law is a common feature in disordered materials [50], spin glasses, polymers. In a CDW system, the disorder would be due to random distribution of impurities.

One should note that relaxation effects are found in the Ohmic resistance. The Ohmic resistance depends on the past thermal and electrical history of the sample (see in Ref. [14e]).

3.5 ComplementARY TECHNiQues. - A detailed understanding of the $\mathrm{CDW}$ transport requires also local methods of investigation. Nuclear magnetic resonance has been proved to be an extremely useful microscopic tool to probe CDW motion via the induced motional narrowing effect $[38,39]$. While
NMR experiments involve intrinsic probes $(\mathrm{Nb}$ in $\mathrm{NbSe}_{3}, \mathrm{Rb}$ in $\mathrm{Rb}_{0.3} \mathrm{MoO}_{3}$ ), electron paramagnetic resonance and Mössbauer spectroscopy involve extrinsic probes, $\mathrm{Mo}^{5+}$ defects in $\mathrm{K}_{0.3} \mathrm{MoO}_{3}$ and ${ }^{57} \mathrm{Fe}$ respectively. These experiments have shed recently some light on the nature of the defects involved in the pinning of the CDW [51].

The consequences of the CDW motion on the mechanical properties of the host crystal have been reported in the case of $\mathrm{NbSe}_{3}$ and $\mathrm{TaS}_{3}$. In particular, a small decrease of the Young modulus is found when the CDW is sliding [52]. Ion-channelling technique has also shed some light on the metastability of the $\mathrm{CDW}$ in $\mathrm{K}_{0.3} \mathrm{MoO}_{3}$ [53].

The elementary excitations of the CDW condensate, namely the phase excitation (phason) and amplitude excitation (amplitudon) have been investigated by optical and inelastic neutron scattering [55] experiments. Since the blue bronze can be grown as large single crystals it is well adapted for neutron experiments.

Direct visualization of CDW domains with and without a current in the sample would be extremely helpful in elucidating the CDW transport mechanism. In this context, dark field electron microscopy experiments in $\mathrm{NbSe}_{3}$ and $\mathrm{TaS}_{3}$ have been reported [56], but no clear conclusions can be drawn from these experiments.

\section{Theoretical models.}

While it is well accepted that the observed non linear transport properties are due to the collective motion of a charge density wave the detailed explanation is still controversial ; in particular, the origin of the periodic voltage oscillations (bulk effect or local under the contacts) is not yet fully elucidated. It is not yet clear whether the CDW moves as a whole as proposed by Fröhlich or by generation of « discommensurations » or phase slips. We discuss below some recent theoretical approaches.

4.1 Classical Rigid MODEL. - In this model $[5,14]$, the response of the CDW to an external fields is that of an harmonic oscillator. A good fit of the high frequency conductivity is obtained in assuming that the oscillator is overdamped. The CDW is analogous to a particle moving in a sinusoidal potential of period $\lambda$ which represents the effective potential due to the impurities ( $\lambda$ being the period of the CDW). To this potential a linear ramp with a slope porportional to $E$ is superimposed. The particle accelerates and slows down in this potential. This leads to a time dependent current with frequency proportional to the dc current. This model explains the field and frequency dependence of the conductivity and the narrow band noise but does not describe satisfactorily the detailed form of the $V-I$ curves nor the broad band noise and hysteresis. 
4.2 Deformable models. - To remove the divergence of the differential conductivity found in the classical model, it is necessary to incorporate the internal degrees of freedom of the CDW. The deformable model of Fukuyama, Lee and Rice [57] takes into account the impurity induced deformation of an incommensurate CDW. The phase of the CDW adjusts locally to minimize the sum of the elastic energy of the CDW and the impurity pinning energy. These authors define a domain size inside which the phase is coherent. From experimental data, the size of a domain is macroscopic (a few microns). A great number of experimental results indicate that the internal deformation of the CDW is essential in understanding the glassy behaviour of the CDW.

In recent deformable models metastable states have been studied in details [44] by numerical simulations. More recently, a quasi-commensurate $\mathrm{CDW}$ has been considered in close analogy with the mechanical deformation of crystals [58]. The CDW is not rigid; it is elastically displaced at low fields and CDW defects (« discommensurations" or dislocations of the phase) are pinned by impurities. At threshold, CDW defects are nucleated and begin to move leading to a permanent plastic flow which is ascribed to the CDW current. Similarities with the crystal plasticity and "Portevin-Le Chatelier » effect, where a coupling between lattice dislocations and impurities is involved, have been discussed.

4.3 VORTEX MODEL. - In this picture [40] the periodic voltage oscillations at constant current is due to the creation of phase vortices at the interfaces where the CDW has a discontinuity (near the electrical contacts). At the contacts, the velocity of the CDW drops abruptly to zero and carriers condensed in the CDW become normal carriers. This carrier conversion process occurs by periodic nucleation of phase vortices which move transverse to the motion of the $\mathrm{CDW}$.

4.4 QUANTUM MODEL. - In a very different approach, Bardeen [59] has suggested that quantum effects are essential in the dynamics of a CDW. He has proposed that the CDW can tunnel across potential barriers before depinning occurs. The small periodic potential leads to a small gap across which the CDW is excited by the electric field. This model is analogous to the Zener tunnelling in semiconductors. Introducing a finite coherence length $l$, this model gives a threshold $E_{\mathrm{t}}$ and the conductivity obeys the law $\sigma \alpha\left(1-E_{\mathrm{t}} / E\right) \exp -$ $\left(E_{0} / E\right)$ which reproduces satisfactority experimental results in some cases. This model provides a good fit to various ac-dc coupling experiments (see Ref. [14e]).

\section{Conclusion.}

Since the discovery in $\mathrm{NbSe}_{3}$ of an entirely new form of conduction in solids due to the motion of a charge density wave considerable progress has been made in the understanding of the associated non linear transport. Detailed studies of this unusual conduction process have revealed a wealth of phenomena of great complexity. The fascinating CDW transport properties are now observed in three different classes of materials. $\mathrm{NbSe}_{3}$ is the unique system which remains metallic at low temperature. Up to now, no widespread agreement exists on the origin of the coherent voltage oscillations which appear spontaneously above threshold. Among the variety of phenomena observed in these CDW materials, they are considered as one of the clearest signature of CDW motion. The role of interactions between lattice defects and CDW defects (discommensurations or phase dislocations) which seems preponderant in the case of the blue bronze as well as metastability phenomena, have to be explored further. Sophisticated methods of investigation have emerged; local methods seem promising. On the other hand, it would be interesting to find other classes of materials showing charge density wave transport.

\section{Acknowledgments.}

The author wish to thank C. Schlenker, P. Monceau, B. K. Chakraverty, D. Feinberg, A. Janossy, G. Mihaly and many other colleagues for numerous and fruitful discussions.
[1] Wilson, J. A., Di Salvo, F. J., Mahajan, S., $A d v$. Phys. 24 (1975) 117.

[2] Schlenker, C., Dumas, J., Escribe-Filippini, C., Guyot, H., Marcus, J., Fourcaudot, G., Philos. Mag. B 52 (1985) 643 ;

Escribe-FilipPini, C., Almairac, R., Ayroles, R., Roucau, C., Konate, K., Marcus, J., Schlenker, C., Philos. Mag. B 50 (1984) 321.

[3] Ganne, M., Dion, M., Boumaza, A., Tournoux, M., Solid State Commun. 59 (1986) 137 ;
Ramanujachary, K. V., Collins, B. T., GreenBLATT, M., WASZCZAK, J. V., Ibid. 59 (1986) 645.

[4] Friedel, J., Jerome, D., Contemp. Phys. 33 (1982) 583.

[5] For a review see : Monceau, P. in Electronic Properties of Inorganic Quasi-One-Dimensional Compounds, part II, Ed. by Monceau P. (Reidel, Dordrecht) 1985, p. 139. 
[6] For a review see : Schlenker, C., Dumas, J. in Crystal Chemistry and Properties of Materials with Quasi-One-Dimensional structures Ed. by Rouxel J. (Reidel, Dordrecht), 1986, p. 135 ;

Fleming, R. M., Synth. Metals 13 (1986) 241.

[7] Ganne, M., Boumaza, A., Dion M., Dumas, J., Mat. Res. Bull. 20 (1985) 1297 ;

Collins, B. T., Ramanujachary, K. V., GreenBlatt, M., WaszczaK, J. V., Solid State Commun. 56 (1985) 1023.

[8] PeIERls, R. E., Quantum Theory of Solids (Clarendon, Oxford) 1953, p. 108.

[9] Fröhlich, H., Proc. Roy. Soc. A 223 (1954) 296.

[10] Monceau, P., Ong, N. P., Portis, A. M., Meerschaut, A., Rouxel, J., Phys. Rev. Lett. 37 (1976) 602.

[11] Sambongi, T., Tsutsumi, K., Shiozaki, Y., Yamamoto, M., Yamaya, K., Abe, Y., Solid State Commun. 22 (1977) 729.

[12] Wang, Z. Z., Saint-Lager, M. C., Monceau, P., Renard, M., Gressier, P., Meerschant, A., Guemas, L., Rouxel, J., Solid State Commun. 46 (1983) 325.

[13] Wang, Z. Z., Monceau, P., Renard, M., GresSier, P., Guemas, L., Meerschant, L., Solid State Commun. 47 (1983) 439.

[14] For a review see for example : a) GUNER, G., ZetTl, A., Phys. Rep. 119 (1985) 117 ;

b) J. Physique Colloq. 44 (1983) C3 ;

c) Int. Conf. on Synthetic Metals, Mol. Crystals Liq. Crystals 121 (1985);

d) Int. Conf. on Synth. Metals, Kyoto 1986, Synth. Metals (to be published);

e) Charge Density Waves in Solids, 217 of Lecture Notes in Physics, ed. by Hutiray Gy and Solyom J. (Springer, Berlin 1985) ;

f) Yamada Conf. on Physics and Chemistry of QuasiOne-Dimensional Conductors (Japan, 1986) to be published in Physica B ;

g) Gill, J. C., Contemp. Phys. 27 (1986) 37 ;

h) Low Dimensional Conductors and Superconductors, NATO-ASI, Aug. 1986, to be published.

[15] Toombs, G. A., Phys. Rep. C 40 (1978) 181.

[16] Lacoe, R. C., Schultz, H. J., Jerome, D., BeChgaArd, K., Johansen, I., Phys. Rev. Lett. 55 (1985) 2351.

[17] Coleman, R. V., Drake, B., Hansma, P. H., Slough, G., Phys. Rev. Lett. 55 (1985) 394.

[18] Fleming, R. M., Moncton, D. E., AXe, J. D., Brown, G. S., Phys. Rev. B 30 (1984) 1877 ;

Hodeau, J. L., Marezio, M., Roucau, C., AyROLES, R., MEerschaUt, A., ROUXEL, J., Monceau, P., J. Phys. C 11 (1978) 4117.

[19] Wang, Z. Z., Salva, H., Monceau, P., Renard, M., Roucau, C., Ayroles, R., Levy, F., Guemas, Z., Meerschaut, A., J. Physique Lett. 44 (1983) L-1311.

[20] Roucau, C., J. Physique Colloq. 44 (1983) C3-1725.

[21] Gressier, P., Meerschaut, A., Guemas, A., Rouxel, J., Monceau, P., J. Physique Colloq. 44 (1983) C3-1741;

Roucau, C., Ayroles, R., Gressier, P., Meerschaut, A., J. Phys. 17 (1984) 2993.
[22] Meerschaut, A., Gressier, P., Guemas, L., Rouxel, J., J. Solid State Chem. 51 (1984) 307.

[23] Pouget, J. P., Kagoshima, S., Schlenker, C., Marcus, J., J. Physique Lett. 44 (1983) L-113.

[24] Chen, Ch., Schneemeyer, L. F., Fleming, R. M., Phys. Rev. B 29 (1984) 3765.

[25] Meerschaut, A., Rouxel, J., J. Less Common Metals 39 (1975) 197.

[26] Chaussy, J., Haen, P., Lasjaunias, J. C., MonCEAU, P., WAysand, G., Waintal, A., Meerschaut, A., Molinie, P., Rouxel, J., Solid State Commun. 20 (1976) 759.

[27] Ong, N. P., Monceau, P., Phys. Rev. B 16 (1977) 3443.

[28] Salva, A., Wang, Z. Z., Monceau, P., Richard, J., Renard, M., Philos. Mag. B 49 (1984) 385.

[29] Meerschaut, A., Guemas, L., Rouxel, J., J. Solid State Chem. 36 (1981) 118.

[30] Greenblatt, M., Mc Carrol, W. H., Neifeld, R., Croft, M., WaszczaK, J. V., Solid State Commun. 51 (1984) 671.

[31] Wold, A., Kunnmann, W., Arnott, R. J., FerRETTI, A., Inorg. Chem. 3 (1964) 545.

[32] Bouchard, G. H., Perlstein, J. H., Sienko, M. J., Inorg. Chem. 6 (1967) 1682.

[33] Dumas, J., Schlenker, C., Marcus, J., Buder, R., Phys. Rev. Lett. 50 (1983) 757.

[34] Ghedira, M., Chenavas, J., Marezio, M., MarCUS, J., J. Solid State Chem. 57 (1985) 300.

[35] Fleming, R. M., Grimes, C. C., Phys. Rev. Lett. 42 (1979) 1423.

[36] Fleming, R. M., Moncton, R. E., Exe, J. D., Brown, G. S., Phys. Rev. B 30 (1984) 1877.

[37] Monceau, P., Richard, J., Renard, M., Phys. Rev. B 25 (1982) 931 ;

Richard, J., Monceau, P., Renard, M., Ibid. B 25 (1982) 948.

[38] Ross, J. H., Wang, Z., Slichter, C. P., Phys. Rev. Lett. 56 (1986) 663.

[39] Nomura, K., Kume, K., Sato, M., J. Phys. C 19 (1986) L289 ;

Segransan, P., Janossy, A., Berthier, C, MarCus, J., Butaud, P., Phys. Rev. Lett. 56 (1986) 1854.

[40] Verma, G., Ong, N. P., Phys. Rev. B 40 (1984) 2928.

[41] Brown, S. E., Janossy, A., Gruner, G., Phys. Rev. B 31 (1985) 6869.

[42] Janossy, A., Mihaly, G., Pekker, S., Roth, Solid State Commun. to be published.

[43] Brown, S. E., Mihaly, L., Phys. Rev. Lett. 55 (1985) 742.

[44] Littlewood, P. B., Phys. Rev. B 33 (1986) 6694 and references therein.

[45] Gill, J. C., Solid State Commun. 39 (1981) 1203.

[46] TAMEgAI et al. in reference [14e].

[47] Fleming, R. M., DunN, R. G., SChneemeyer, L. F., Phys. Rev. B 31 (1985) 4099.

[48] Cava, R. J., Fleming, R. M., Rietman, E. A., Dunn, R. G., SCHNEemeyer, L. F., Phys. Rev. Lett. 53 (1984) 1677.

[49] Kriza, G., Mihaly, G., Phys. Rev. Lett. 56 (1986) 2529. 
[50] Ngai, K. L., Rendell, R. W., in Handbook of Conducting Polymers ed. by Stokheim T. A. (Dekker) 1986, vol. 2, p. 967.

[51] Dumas, J., Schlenker, C., Veuillen, J. Y., Chevalier, R., Marcus, J., Cinti, R., Al KHOURY, E., in reference [14d].

[52] See for example BRILL, J. W., in reference [14e].

[53] Dumas, J., Schlenker, C., Beauchene, P., FilipPINI, C., BUDER, R., DAUDIN, B., in reference [14d].

[54] Travaglini, G., Wachter, P., Phys. Rev. B 30 (1984) 1971.
[55] Filippini, C., Pouget, J. P., Hennion, B., Sato, M., in Ref. [14d].

[56] Fung, K. K., Steeds, J. W., Phys. Rev. Lett. 45 (1980) 1696.

[57] Funuyama, H., Lee, P. A., Phys. Rev. B 17 (1977) 535 ;

Lee, P. A., Rice, T. M., Phys. Rev. B 19 (1979) 3970.

[58] Dumas, J., Feinberg, D., Europhys. Lett. 2 (1986) 555.

[59] Bardeen, J., Phys. Rev. Lett. 45 (1980) 1978. 